\title{
Nanomechanics of Bidentate Thiolate Ligands on Gold Surfaces
}

\author{
Martin E. Zoloff Michoff, ${ }^{*}$ Jordi Ribas-Arino, ${ }^{\dagger}$ and Dominik Marx \\ Lehrstuhl für Theoretische Chemie, Ruhr-Universität Bochum, 44780 Bochum, Germany
}

(Received 24 October 2014; published 17 February 2015)

\begin{abstract}
The effect of the chain length separating sulfur atoms in bidentate thiols attached to defective gold surfaces on the rupture of the respective molecule-gold junctions has been studied computationally. Thermal desorption always yields cyclic disulfides. In contrast, mechanochemical desorption leads to cyclic gold complexes, where metal atoms are extracted from the surface and kept in tweezer-like arrangements by the sulfur atoms. This phenomenon is rationalized in terms of directional mechanical manipulation of $\mathrm{Au}-\mathrm{Au}$ bonds and $\mathrm{Au}-\mathrm{S}$ coordination numbers. Moreover, the flexibility of the chain is shown to crucially impact on the mechanical strength of the junction.
\end{abstract}

DOI: 10.1103/PhysRevLett.114.075501

PACS numbers: 62.25.-g, 68.43.Bc, 68.43.Vx, 73.22.-f

Thiolate-gold interfaces have been intensely studied for many decades using a wide array of experimental and computational methodologies $[1,2]$. The pronounced interest in these particular hybrid molecule-metal junctions and interfaces is due to a multitude of potential applications such as tailoring the properties of surfaces [3-7], chemical anchors for molecular electronics applications [8-10], or coating agents for the stabilization of gold nanoparticles $[11,12]$. Only lately, it has been realized that the consideration of the mechanical properties of these moleculemetal hybrids becomes a crucial design factor [13-24] for nanoscale devices.

In recent years, the interest in multidentate thiolate ligands adsorbed on metallic surfaces has grown rapidly because of the search for an enhanced interaction [25]. Specifically, thioctic acid (TA), a bidentate thiolate, has been widely used as the anchor for polyethylenglycol (PEG) chains to "PEGylate" gold nanoparticles [26-31]. This ligand provides enhanced stability to gold nanoparticles under a wide range of conditions with respect to their monothiolated counterparts [28-30]. Selfassembled monolayers (SAMs) of aromatic dithiols on gold have been shown to be more robust than the analogous normal alkanothiolate and aromatic thiolate SAMs [32,33].

Despite the increasing interest in exploiting multidentate thiols for functionalization, all experimental and theoretical studies performed so far have dealt only with the structure and thermodynamic stability of these adsorbates. Information about how such multidentate SAMs or junctions would respond to external stress is conspicuously absent, whereas monothiol-based interfaces and point contacts have been intensely studied [13,20,34-41]. To the best of our knowledge, only one paper dealing with the mechanical rupture of a dithiolate linkage to gold has been reported [42]. Interestingly, the measured force required to remove a single TA molecule from the gold substrate resulted in an about 3.4 times smaller rupture force compared to that of a simple Au-S bond. This suggests that SAMs of multidentate thiols may be less stable under tensile stress than anticipated from their thermal properties via thermal desorption experiments and computed binding energies. Yet, no explanation of this most puzzling result is available in the literature known to us.

Here, we shed light onto this open topic by investigating, by means of electronic structure calculations, the mechanical desorption of a series of bidentate thiolated ligands adsorbed on a defective gold surface in a one-to-one comparison to thermal desorption; see the Supplemental Material (SM) for computational methods [43]. The anchoring sulfur atoms are separated by a carbon bridge of increasing length from one to four spacer atoms. The molecules studied in this work are ethane-1,1-dithiol (abbreviated by $\mathbf{C} 1$ in the following), butane-2,3-dithiol (C2), pentane-2,4-dithiol (C3), and 2,3-dimethylbutane1,4-dithiol (C4), as depicted by the structures labeled as "open chain dithiols" in Fig. S1. Methyl groups were added at suitable positions as handles to enable mechanical manipulation of the adsorbates.

Our calculations reveal that the preferred thermally activated desorption product is the detachment of the cyclic disulfides. A distinct correlation between the desorption energy and the strain energy of the cyclic products explains these observations. In stark contrast, in the mechanically activated desorption pathway, the $\mathrm{Au}$-Au bonds are preferentially activated instead. This leads to the detachment of one up to three $\mathrm{Au}$ atoms from the metal surface, which are complexed by the bidentate thiolate ligands in tweezer-like structures. The following discussion focuses on the representative system $\mathbf{C 2}$, which has been chosen as our showcase to illustrate the key findings, whereas the supporting data from all other cases have been collected in the SM.

Three products were considered for the thermal desorption process: the corresponding cyclic disulfides as well as cyclic gold complexes containing one or two gold atoms extracted from the surface. The resulting desorption energies $\left(E_{\mathrm{des}}\right)$ are summarized in Table I. It can be clearly 
TABLE I. Desorption energies $\left(E_{\mathrm{des}}\right)$ from the defective $\mathrm{Au}(111)$ surface slab model (see SM) for $\mathbf{C 1}$ to $\mathbf{C} 4$ to yield the corresponding cyclic disulfides (SS) or cyclic gold complexes by extraction of one $(\mathrm{Au})$ or two $(2 \mathrm{Au})$ gold atoms from the surface, energy to form the cyclic disulfides from the corresponding bidentate thiols $\left(-E_{\text {red }}\right)$, RSEs for the cyclic disulfides, and rupture forces $\left(F_{\text {rup }}\right)$ obtained from mechanical desorption (see the SM for definitions). Energies are given in $\mathrm{eV}$ and forces in $\mathrm{nN}$.

\begin{tabular}{lcccccc}
\hline \hline Molecule & $E_{\text {des }}^{\text {SS }}$ & $E_{\text {des }}^{\text {Au }}$ & $E_{\text {des }}^{2 A u}$ & $-E_{\text {red }}$ & RSE & $F_{\text {rup }}$ \\
\hline C1 & 2.49 & 4.56 & 5.92 & 1.04 & 0.86 & 1.98 \\
C2 & 2.17 & 4.03 & 5.26 & 1.09 & 0.88 & 1.25 \\
C3 & 1.76 & 3.74 & 5.33 & 0.54 & 0.35 & 2.05 \\
C4 & 1.37 & 3.14 & 4.99 & 0.26 & 0.22 & 2.01 \\
\hline \hline
\end{tabular}

appreciated that cyclic disulfides are the preferred desorption products. The desorption energy decreases by $\sim 50 \%$ in the order $\mathbf{C 1}>\mathbf{C 2}>\mathbf{C} \mathbf{3}>\mathbf{C 4}$, depending on the length of the carbon bridge between the sulfur sites. Most importantly, the extraction of gold atoms from the surface within a thermal process is significantly disfavored in all cases by approximately $2-3 \mathrm{eV}$.

To rationalize the observed behavior, ring strain energies (RSEs) for the cyclic disulfides were calculated (according to the group equivalent reaction procedure [54]) and are also compiled in Table I. A good correlation was found between RSE and $E_{\text {des }}$ values with the exception of $\mathbf{C 1}$, for which a slight over binding is observed (see the SM for details). The observed trend in the desorption energies can be tracked down to a combination of reactant (adsorbate) destabilization, driven by the tilt angle of the S-C bond with respect to the direction perpendicular to the surface and product (cyclic disulfide) stabilization, governed by the $\mathrm{C}-\mathrm{S}-\mathrm{S}-\mathrm{C}$ torsion angle. For $\mathrm{CH}_{3} \mathrm{~S}$ and for other monodentate thiols adsorbed on $\mathrm{Au}(111)$ surface, the optimal tilt angle is $\sim 60^{\circ}$ (see, e.g., Refs. $[35,55,56]$ ) and is related to the orientation of sulfur orbitals. The tilt angles of the C-S bonds in the adsorbed structures are about $59^{\circ}, 36^{\circ}, 32^{\circ}$, and $43^{\circ}$ for $\mathbf{C 1}, \mathbf{C 2}, \mathbf{C 3}$, and $\mathbf{C 4}$, respectively. As was recently pointed out [57], this reduction in the tilt angle for bidentate thiolates, which is an immediate consequence of the constraints imposed by the rather short carbon bridge between the two $\mathrm{S}$ atoms, can be related to a destabilization of the adsorption of dithiolated anchors on $\mathrm{Au}(111)$. As it is well known, a value of $\sim 90^{\circ}$ is preferred for the C-S-S-C torsion angle in open chain dialkyl disulfides [58]. For the cyclic disulfides that are obtained as thermal desorption products, this value increases in the order $\mathbf{C 2}\left(19^{\circ}\right)<$ $\mathbf{C 3}\left(46^{\circ}\right)<\mathbf{C 4}\left(58^{\circ}\right)$, and thus, thermal desorption of $\mathbf{C 4}$ enormously is favored.

A typical pathway for the mechanical desorption of these dithiolated ligands is illustrated in Fig. 1(a) for C2. The extension distance $(D)$ is the control parameter in these pulling simulations (see SM). It is noted in passing that this isometric approach corresponds to the "quasistatic pulling" limit [18], in stark contrast to dynamical constant-velocity approaches, where the pulling velocity and thus the generated time scales of plastic deformations are unreasonably fast compared to experiment. The action of the mechanical force on the molecules causes the nanojunction to undergo a series
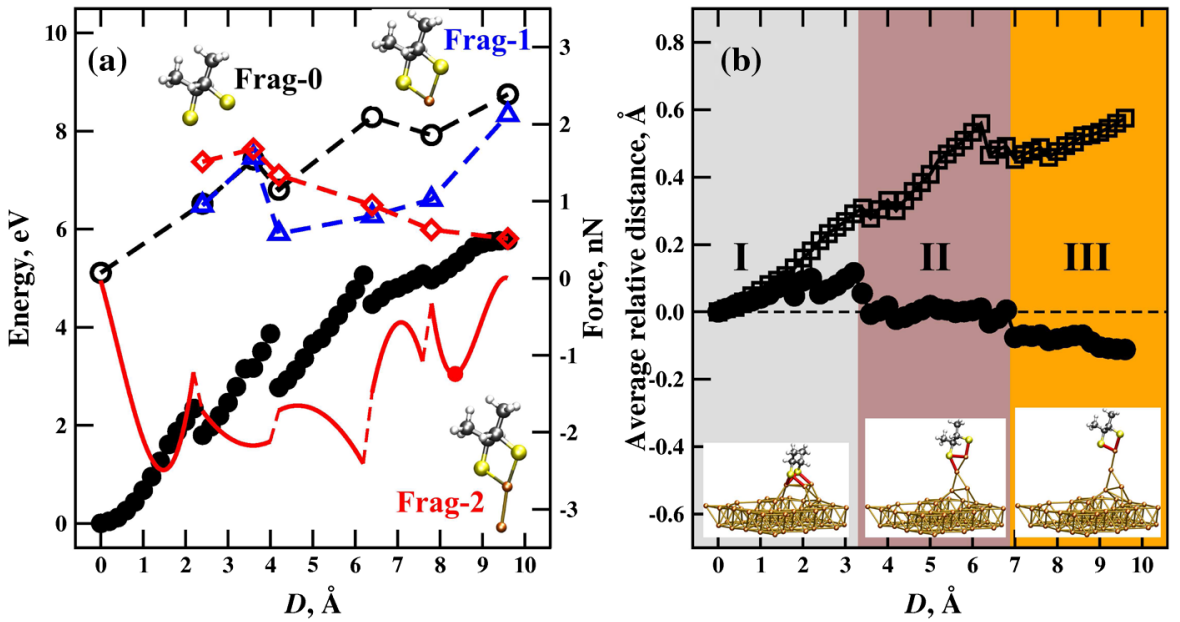

(c)
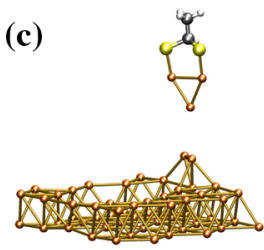

C1
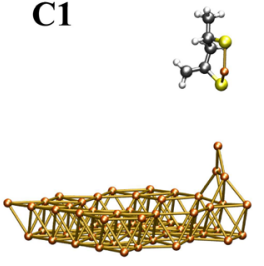

C3

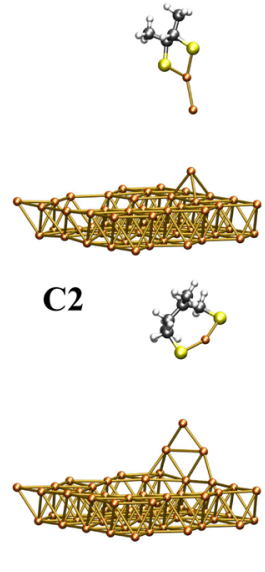

C4

FIG. 1 (color online). (a) Pathway of mechanical desorption of C2 (see Figs. S4, S5, and S6 for the C1, C3, and C4 species, respectively): Total electronic energy along the mechanical desorption pathway (filled black circles), total electronic energy of the fragmentation products Frag-0 (empty black circles), Frag-1 (empty blue triangles), and Frag-2 (empty red diamonds), as illustrated in Fig. S8. The insets illustrate the products (molecule only) for the three fragmentation scenarios at $D=7.8 \AA$. Force versus distance curves for regions of elastic deformation (solid red lines, right axis) are shown; the connecting broken red lines are merely guides to the eye through discontinuous

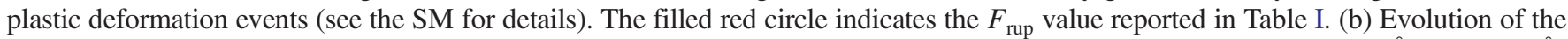
average vertical distance of those Au atoms that are initially in the top layer (empty squares) relative to this distance (7.29 $\AA$ ) at $D=0 \AA$, and average Au-S distance (filled circles) relative to this distance $(2.40 \AA)$ at $D=0 \AA$ along the mechanical desorption pathway. The shaded areas indicate the different Au-S coordination numbers (see text for definition) in the three distinct regimes along the detachment pathway, (I) $2: 2$, (II) $2: 1$, and (III) $1: 1$, whereas the insets are representative snapshots at $D$ values of $2.4,6.4$, and $7.8 \AA$, respectively. (c) Final products for the mechanical desorption of the studied adsorbates. Note that the two bottom gold layers are omitted for clarity. 
of elastic and plastic deformations, leading to the formation of a neck of gold atoms between the molecule and the surface until final breakage of the junction. In stark contrast to what is observed for thermal desorption, the mechanically preferred products are cyclic complexes in which gold atoms are extracted out of the surface [see Fig. 1(c)].

In order to understand this significant difference in behavior between thermal and mechanical desorption, we calculated "vertical fragmentation energies" $\left(E^{\mathrm{frag}}\right)$ at selected configurations along the mechanical desorption pathway. This has been done for a set of distinct fragmentation scenarios in which either the S-Au bonds or some selected $\mathrm{Au}-\mathrm{Au}$ bonds have been broken. All calculated $E^{\text {frag }}$ values are collected in Tables $\mathrm{S} 1$ to S4, and the total energies for the fragmentation products (see SM) are plotted in Fig. 1(a) for C2 being our representative example. Although it is observed that the $E_{0}^{\text {frag }}$ values (for fragmentation at the Au-S bonds) decrease steadily along the desorption pathway, they are always $\sim 3-5 \mathrm{eV}$ above the energy corresponding to mechanical stretching. On the other hand, for the scenario in which two gold atoms are extracted from the surface by the dithiol tweezers, the fragmentation energies $E_{2}^{\text {frag }}$ rapidly decrease upon stretching the nanojunction. At $D=7.8 \AA$, $E_{2}^{\text {frag }}$ is only roughly $1 \mathrm{eV}$ above the energy of the stretching pathway. At rupture, this energy difference vanishes, explaining that the cyclic gold complex C2 in Fig. 1(c) is the preferred mechanical desorption product.

Further insight can be gained by analyzing the evolution of the average Au-S bond distance, the average vertical mean displacement of the Au atoms that are initially located in the adsorption layer, and the coordination number of each $\mathrm{S}$ atom with respect to $\mathrm{Au}$ atoms, $n_{\mathrm{S} \text {-Au }}$, defined as the number of S-Au contacts within a sphere of radius $2.70 \AA$ around each sulfur atom. Our representative C2 scenario depicted in Fig. 1(b) (see Figs. S4, S5, and S6 for C1, C3, and C4, respectively) shows that in the initial stages the $\mathrm{Au}-\mathrm{S}$ bonds are only slightly elongated, which is the elastic regime of mechanical response, but as the surface gets subsequently modified and Au adatoms are created by the mechanical force acting on the molecule-metal junction, i.e., upon entering the plastic regime, the coordination number decreases steadily from 2:2 (i.e., both $\mathrm{S}$ atoms having $n_{\mathrm{S} \text {-Au }}=2$ ) to $1: 1$. This reduction is accompanied with a concurrent decrease of the average $\mathrm{Au}-\mathrm{S}$ bond distance in contrast to the increase observed in the elastic regime. At the same time, Au atoms are continuously lifted from the surface, as evidenced by the continuous increase in the average vertical displacement of the Au atoms. This analysis shows that the external force preferentially activates $\mathrm{Au}-\mathrm{Au}$ bonds over $\mathrm{Au}-\mathrm{S}$ bonds, in line with the calculated $E^{\text {frag }}$ values.

An interesting effect due to the size of the molecule arises when comparing the pulling pathways of $\mathbf{C 2}, \mathbf{C 3}$, and $\mathbf{C 4}$, which are very similar for the three molecules except for the very last stage before final rupture of the nanojunction (see top panel of Fig. 2). At this point, a peculiar Au-S-Au-S bonding pattern that resembles that found for thiols adsorbed on gold surfaces [59] or thiolate-protected gold nanoparticles [60] is formed. This occurs at $D$ values of $6.4 \AA$ (for C2), $6.6 \AA(\mathbf{C 3})$, and $7.2 \AA(\mathbf{C 4})$. When $D$ increases beyond these values, two distinct behaviors are discovered: for $\mathbf{C 2}$, one $\mathrm{S}-\mathrm{Au}$ bond is broken, resulting in both $\mathrm{S}$ atoms being bonded to the terminal $\mathrm{Au}$ atom of the wire, whereas for the other two molecules, further stretching causes Au-Au bond breaking. In this last case, this particular bonding motif is maintained so that one $\mathrm{S}$ atom remains bonded to two gold atoms, bridging the molecule to the surface. Although the final products are all similar, i.e., cyclic gold complexes, the different plastic response of these molecule-metal junctions due to tensile force leads to a distinctly different structure of the molecule-surface junction. This, in turn, substantially affects the mechanical strength of the system, as evidenced by the $F_{\text {rup }}$ values in Table I.

Indeed, the different nanomechanical behavior can be related to the flexibility of the spacer bridge between the $\mathrm{S}$ atoms. This is best illustrated by the variation of the $\mathrm{S}-\mathrm{Au}-\mathrm{S}$ angle within the tweezer-like arrangement. A linear $\mathrm{S}-\mathrm{Au}-\mathrm{S}$ bond is thermodynamically favored in the case of monothiols [61]. At the initial point, the S-Au-S angle increases in the sense $\mathbf{C 2}\left(98^{\circ}\right)<\mathbf{C 4}\left(110^{\circ}\right)<\mathbf{C} 3\left(119^{\circ}\right)$. For the case of $\mathbf{C 2}$, the additional strain created by stretching

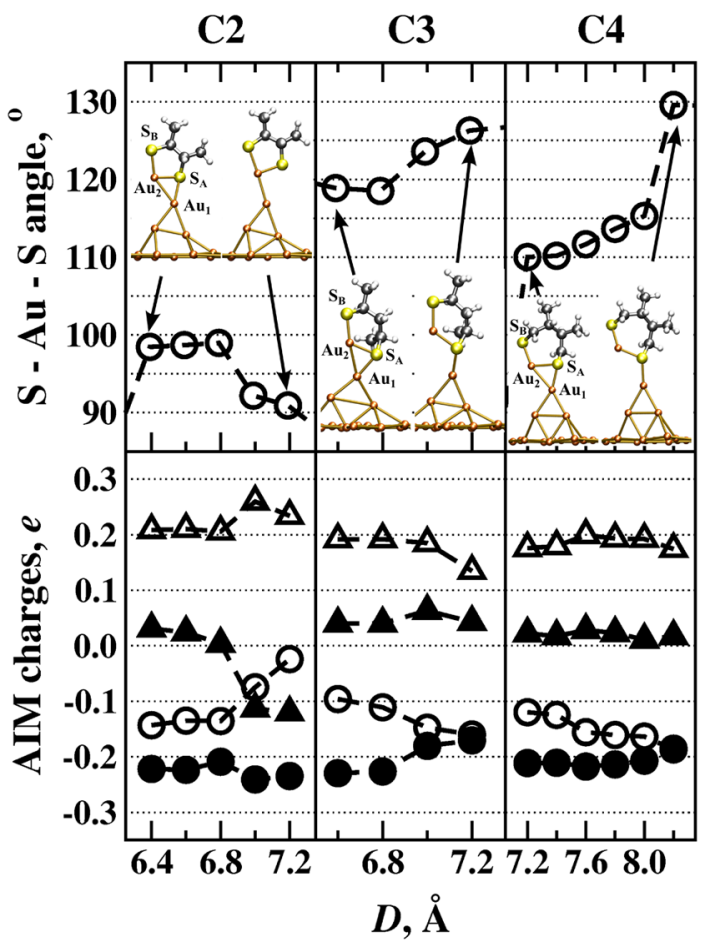

FIG. 2 (color online). (Top) S-Au-S angle for parts of the mechanical desorption pathways (as parametrized by $D$ ) of $\mathbf{C 2}$, C3, and C4. The insets introduce the atom labels and illustrate the relevant structural changes at $D=6.4 \AA$ and $7.2 \AA$ (C2), $6.6 \AA$ and $7.2 \AA$ (C3), $7.2 \AA$ and $8.2 \AA$ (C4). (Bottom) Atoms-inmolecules charges for $\mathrm{S}_{A}$ (empty circles), $\mathrm{S}_{B}$ (filled circles), $\mathrm{Au}_{1}$ (empty triangles), and $\mathrm{Au}_{2}$ (filled triangles) for the same part of the mechanical desorption pathways of $\mathbf{C 2}, \mathbf{C 3}$, and $\mathbf{C 4}$ (see top insets for atom labeling). 
the structure at $D=6.6 \AA$ causes a reduction of the S-Au-S angle to $\sim 91^{\circ}$. The $\mathrm{S}$ atom that is initially coordinated to two gold atoms is forced to reduce its coordination number to one. In contrast, for the other two larger molecules, the $\mathrm{S}$-Au-S angle is found to increase (to $\sim 126^{\circ}$ for $\mathbf{C 3}$ and to $\sim 129^{\circ}$ for $\mathbf{C 4}$ ) at the expense of expanding the C-C-C angles in these molecules. This additional flexibility is missing in C2 due to the shorter bridge. The resulting force-induced deformation of the spacer chain enables the aforementioned $\mathrm{S}$ atom to keep its coordination number of two all the way until final rupture of the junction.

The structural analysis is corroborated by electronic structure analysis. "Atoms-in-molecules" charges [62] were calculated along the different stretching pathways and are plotted in the lower panels of Fig. 2. Close inspection shows that there is a correlation between the observed behavior and the charge transfer that occurs between the $\mathrm{S}$ and relevant $\mathrm{Au}$ atoms (see the configuration snapshots in Fig. 2 for atom labels). For C2, the stretching of the junction induces a charge transfer $S_{A} \rightarrow A u_{1}$ so that $S_{A}$, which is initially negatively charged, becomes less negative and $\mathrm{Au}_{1}$, initially slightly positive, becomes negatively charged. This weakens the $\mathrm{S}_{A}-\mathrm{Au}_{1}$ bond, which finally breaks. On the other hand, for $\mathbf{C 3}$ and $\mathbf{C 4}$, the charge transfer occurs in the sense $\mathrm{S}_{B} \rightarrow$ $\mathrm{S}_{A}$ through $\mathrm{Au}_{2}$. As a consequence, $\mathrm{S}_{A}$ becomes more negative, thus strengthening the $\mathrm{S}_{A}-\mathrm{Au}_{1}$ bond. The weakening and strengthening of the relevant bonds along the stretching pathway is also reflected in the calculated Mulliken Overlap Population values [63], being a complementary approach, as summarized in Table S5.

Projected density of states (pDOS) analysis of the stretched nanojunctions shows that there is a significant difference in the bonding picture between $\mathbf{C 2}$ and the other two larger molecules, which is illustrated in Fig. 3. The main bonding interactions are due to $\mathrm{S} 3 p$ and $\mathrm{Au} 5 d$ bands, as has already been shown to be the case in, for instance, thiolateprotected gold nanoclusters [61]; detailed analyses of Au $s$ and $p$ orbitals show that their total contribution never exceeds $20 \%$, although the s contributions to bonding tend to increase systematically upon stretching the nanojunction (see the SM for details). For the larger molecules, the pDOS analysis reveals that there is significant bonding between $S_{B}$ with both $\mathrm{Au}_{1}$ and $\mathrm{Au}_{2}$, whereas for $\mathbf{C} \mathbf{2}$ the spatial constraint between the $\mathrm{S}$ atoms prevents the $\mathrm{S} 3 p$ orbitals to have an optimal orientation for an efficient interaction with the two $\mathrm{Au}$ atoms at the same time. This molecular orbital-based explanation is clearly supported by inspecting selected molecular orbitals that are depicted as insets in Fig. 3.

In short, we have shown that the thermal and mechanical desorption pathways of dithiolate ligands adsorbed on gold surfaces lead to distinctly different products. This difference can be understood in terms of the directional mechanical manipulation of $\mathrm{Au}-\mathrm{Au}$ bonds and the coordination number of the sulfur anchoring sites with respect to gold atoms. The distance constraint imposed by the carbon spacer bridge is found to have a dramatic effect on the

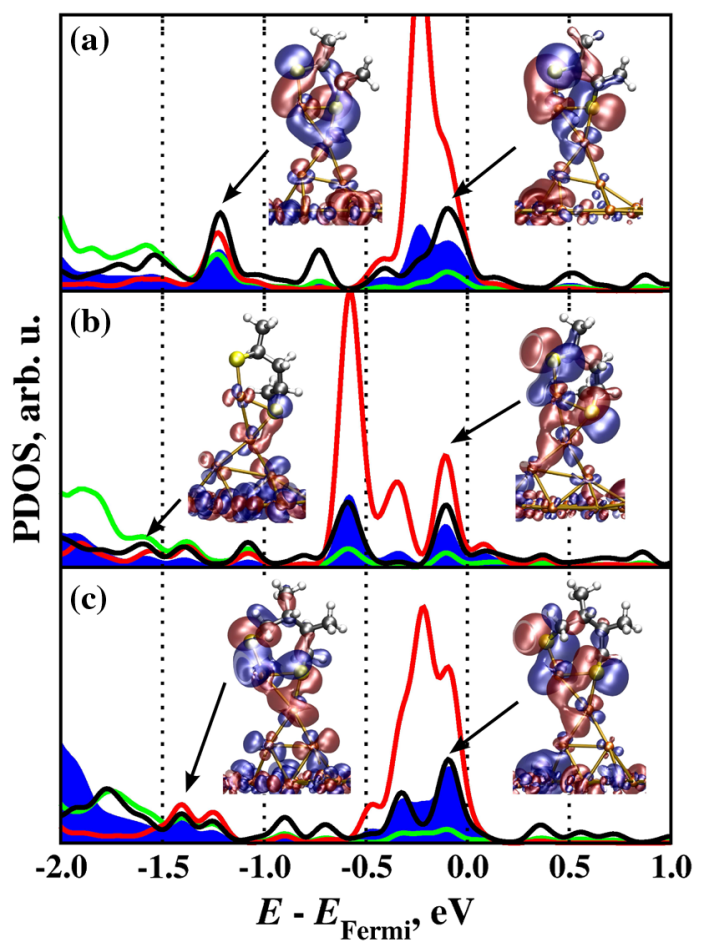

FIG. 3 (color online). Projected density of states for (a) C2 at $D=6.4 \AA$, (b) C3 at $D=6.6 \AA$, and (c) C4 at $D=7.2 \AA$. The depicted orbitals are $3 p$ for $\mathrm{S}_{A}$ (black line) and $\mathrm{S}_{B}$ (red line) and $5 d$ for $\mathrm{Au}_{1}$ (blue shaded area) and $\mathrm{Au}_{2}$ (green line). The labeling of the atoms corresponds to that introduced in Fig. 2, and the insets illustrate relevant molecular orbitals corresponding to the pDOS states indicated by the arrows (isosurface representation at $0.00015 e \mathrm{Bohr}^{-3}$ ).

mechanical strength of these molecule-metal nanojunctions depending on the length of the bridge. This phenomenon has been demonstrated to originate from the different bonding pattern that is observed when the Au-S-Au-S bonding motif arises as evidenced by electronic structure analysis. Given these insights, what remains to be seen in the future is to what extent solvent effects might affect the rupture scenario and thus the mechanical strength of nanojunctions.

M.E.Z.M. and D. M. gratefully acknowledge financial support by DAAD (Fellowship) and DFG (Reinhart Koselleck Grant No. MA 1547/9), respectively. The simulations were carried out on the Cray platform at HLRS (Stuttgart) and at BovilaB@RUB (Bochum).

*martin.zoloff@theochem.ruhr-uni-bochum.de

Permanent address: INFIQC-CONICET, Departamento de Matemática y Física, Facultad de Ciencias Químicas, Univesidad Nacional de Córdoba, Córdoba, Argentina.

Present address: Departament de Química Física and IQTCUB, Facultat de Química, Universitat de Barcelona, Barcelona, Spain.

[1] E. Pensa, E. Cortés, G. Corthey, P. Carro, C. Vericat, M. H. Fonticelli, G. Benítez, A. A. Rubert, and R. C. Salvarezza, Acc. Chem. Res. 45, 1183 (2012). 
[2] H. Häkkinen, Nat. Chem. 4, 443 (2012).

[3] A. Uysal, B. Stripe, B. Lin, M. Meron, and P. Dutta, Phys. Rev. Lett. 107, 115503 (2011).

[4] H. Hamoudi, S. Neppl, P. Kao, B. Schüpbach, P. Feulner, A. Terfort, D. Allara, and M. Zharnikov, Phys. Rev. Lett. 107, 027801 (2011).

[5] A. T. Zayak, Y.S. Hu, H. Choo, J. Bokor, S. Cabrini, P. J. Schuck, and J. B. Neaton, Phys. Rev. Lett. 106, 083003 (2011).

[6] C. Vericat, M. E. Vela, G. Benitez, P. Carro, and R. C. Salvarezza, Chem. Soc. Rev. 39, 1805 (2010).

[7] F.-S. Li, W. Zhou, and Q. Guo, Phys. Rev. B 79, 113412 (2009).

[8] A. Saffarzadeh, F. Demir, and G. Kirczenow, Phys. Rev. B 89, 045431 (2014).

[9] R. J. C. Batista, P. Ordejón, H. Chacham, and E. Artacho, Phys. Rev. B 75, 041402 (2007).

[10] N. J. Tao, Nat. Nanotechnol. 1, 173 (2006).

[11] P. Zhao, N. Li, and D. Astruc, Coord. Chem. Rev. 257, 638 (2013).

[12] X. Chen, M. Strange, and H. Häkkinen, Phys. Rev. B 85, 085422 (2012).

[13] D. Krüger, H. Fuchs, R. Rousseau, D. Marx, and M. Parrinello, Phys. Rev. Lett. 89, 186402 (2002).

[14] M. Konôpka, R. Turanský, J. Reichert, H. Fuchs, D. Marx, and I. Štich, Phys. Rev. Lett. 100, 115503 (2008).

[15] M. Konôpka, R. Turanský, M. Dubecký, D. Marx, and I. Štich, J. Phys. Chem. C 113, 8878 (2009).

[16] P. Vélez, S. A. Dassie, and E. P. M. Leiva, Phys. Rev. B 81, 235435 (2010).

[17] I. Franco, G. C. Solomon, G. C. Schatz, and M. A. Ratner, J. Am. Chem. Soc. 133, 15714 (2011).

[18] J. Ribas-Arino and D. Marx, Chem. Rev. 112, 5412 (2012).

[19] J. Zhou, C. Guo, and B. Xu, J. Phys. Condens. Matter 24, 164209 (2012).

[20] P. Seema, J. Behler, and D. Marx, Phys. Chem. Chem. Phys. 15, 16001 (2013).

[21] S. A. Paz, M. E. Z. Michoff, C. F. A. Negre, J. A. Olmos-Asar, M. M. Mariscal, C. G. Sánchez, and E. P. M. Leiva, Phys. Chem. Chem. Phys. 15, 1526 (2013).

[22] B. M. Szyja, H. C. Nguyen, D. Kosov, and N. L. Doltsinis, J. Mol. Model. 19, 4173 (2013).

[23] H. C. Nguyen, B. M. Szyja, and N. L. Doltsinis, Phys. Rev. B 90, 115440 (2014).

[24] D.-L. Bao, R. Liu, J.-C. Leng, X. Zuo, Y. Jiao, Z.-L. Li, and C.-K. Wang, Phys. Lett. A 378, 1290 (2014).

[25] P. Chinwangso, A. C. Jamison, and T. R. Lee, Acc. Chem. Res. 44, 511 (2011).

[26] K. Susumu, H. T. Uyeda, I. L. Medintz, T. Pons, J. B. Delehanty, and H. Mattoussi, J. Am. Chem. Soc. 129, 13987 (2007).

[27] W. Eck, G. Craig, A. Sigdel, G. Ritter, L. J. Old, L. Tang, M. F. Brennan, P. J. Allen, and M. D. Mason, ACS Nano 2, 2263 (2008).

[28] G. Zhang, Z. Yang, W. Lu, R. Zhang, Q. Huang, M. Tian, L. Li, D. Liang, and C. Li, Biomaterials 30, 1928 (2009).

[29] B. C. Mei, E. Oh, K. Susumu, D. Farrell, T. J. Mountziaris, and H. Mattoussi, Langmuir 25, 10604 (2009).

[30] E. Oh, K. Susumu, R. Goswami, and H. Mattoussi, Langmuir 26, 7604 (2010).

[31] E. Oh, K. Susumu, V. Jain, M. Kim, and A. Huston, J. Colloid Interface Sci. 376, 107 (2012).

[32] N. Garg and T. R. Lee, Langmuir 14, 3815 (1998).
[33] N. Garg, E. Carrasquillo-Molina, and T. R. Lee, Langmuir 18, 2717 (2002).

[34] C. B. Gorman, R. L. Carroll, Y. He, F. Tian, and R. Fuierer, Langmuir 16, 6312 (2000).

[35] D. Krüger, H. Fuchs, R. Rousseau, D. Marx, and M. Parrinello, J. Chem. Phys. 115, 4776 (2001).

[36] J. M. Keel, J. Yin, Q. Guo, and R. E. Palmer, J. Chem. Phys. 116, 7151 (2002).

[37] D. Krüger, R. Rousseau, H. Fuchs, and D. Marx, Angew. Chem., Int. Ed. Engl. 42, 2251 (2003).

[38] B. Xu and N. J. Tao, Science 301, 1221 (2003).

[39] M. Konôpka, R. Rousseau, I. Štich, and D. Marx, J. Am. Chem. Soc. 126, 12103 (2004).

[40] F. Chen, A. Zhou, and H. Yang, Appl. Surf. Sci. 255, 6832 (2009).

[41] Y. Xue, X. Li, H. Li, and W. Zhang, Nat. Commun. 5, 4348 (2014).

[42] K. C. Langry, T. V. Ratto, R. E. Rudd, and M. W. McElfresh, Langmuir 21, 12064 (2005).

[43] See Supplemental Material http://link.aps.org/supplemental/ 10.1103/PhysRevLett.114.075501 for computational details, validation of the calculations, precise definitions of how certain quantities were calculated, tables compiling all the calculated data and additional figures for the systems not shown in the manuscript. It also includes Refs. [44-53].

[44] J. P. Perdew, K. Burke, and M. Ernzerhof, Phys. Rev. Lett. 77, 3865 (1996).

[45] P. Giannozzi et al., J. Phys. Condens. Matter 21, 395502 (2009).

[46] D. Vanderbilt, Phys. Rev. B 41, 7892 (1990).

[47] G. Henkelman, A. Arnaldsson, and H. Jónsson, Comput. Mater. Sci. 36, 354 (2006).

[48] E. Artacho, E. Anglada, O. Diéguez, J. D. Gale, A. García, J. Junquera, R. M. Martin, P. Ordejón, J. M. Pruneda, D. Sánchez-Portal, and J. M. Soler, J. Phys. Condens. Matter 20, 064208 (2008).

[49] N. Troullier and J. L. Martins, Phys. Rev. B 43, 1993 (1991).

[50] N. Troullier and J. L. Martins, Phys. Rev. B 43, 8861 (1991).

[51] Y. H. Jang and W. A. Goddard, J. Phys. Chem. C 112, 8715 (2008).

[52] P. Carro, A. H. Creus, A. Muñoz, and R. C. Salvarezza, Langmuir 26, 9589 (2010).

[53] A. Franke and E. Pehlke, Phys. Rev. B 81, 075409 (2010).

[54] S. M. Bachrach, J. Chem. Educ. 67, 907 (1990).

[55] Y. Yourdshahyan and A. M. Rappe, J. Chem. Phys. 117, 825 (2002).

[56] F. P. Cometto, P. Paredes-Olivera, V. A. Macagno, and E. M. Patrito, J. Phys. Chem. B 109, 21737 (2005).

[57] E. Fertitta, E. Voloshina, and B. Paulus, J. Comput. Chem. 35, 204 (2014).

[58] V. Aquilanti, M. Ragni, A. C. P. Bitencourt, G. S. Maciel, and F. V. Prudente, J. Phys. Chem. A 113, 3804 (2009).

[59] P. Maksymovych, D. C. Sorescu, and J. T. Yates, Phys. Rev. Lett. 97, 146103 (2006).

[60] P. D. Jadzinsky, G. Calero, C. J. Ackerson, D. A. Bushnell, and R. D. Kornberg, Science 318, 430 (2007).

[61] D.-e. Jiang, M. L. Tiago, W. Luo, and S. Dai, J. Am. Chem. Soc. 130, 2777 (2008).

[62] R. F. W. Bader, Atoms in Molecules (Oxford University Press, New York, 1990).

[63] R. S. Mulliken, J. Chem. Phys. 23, 1841 (1955). 